\title{
Heteropterys tomentosa Improves the Endurance Capacity of Skeletal Muscles in Trained Rats
}

\author{
${ }^{1}$ Juliana C. Monteiro Pirovani, ${ }^{2}$ Marcos L. M. Gomes, ${ }^{3}$ Fernando O. Catanho da \\ Silva, ${ }^{4}$ Gerson E. R. Campos, ${ }^{5}$ Heidi Dolder \\ I'Departamento de Ciências Agrárias e Biológicas, Universidade Federal do Espírito Santo, Brasil) \\ ${ }_{2}^{2}$ (Departamento de Biologia Estrutural, Universidade Federal do Triângulo Mineiro, Brasil) \\ ${ }_{3}^{3}$ (Departamento de Bioquímica, Universidade Estadual de Campinas, Campinas, SP, Brasil) \\ ${ }^{4}$ (Universidade Metodista de Piracicaba, Brasil) \\ ${ }^{5}$ (Departamento de Biologia Estrutural e Funcional, Universidade Estadual de Campinas, Brasil)
}

\begin{abstract}
The association of Heteropterys tomentosa infusion with endurance training improves the efficiency of the extracellular matrix remodeling, resulting in more resistant tendons to support high loads from intense muscle contraction. The present study aimed to evaluate the effect of $H$. tomentosa infusion on the skeletal muscles of sedentary and exercised animals. Male Wistar rats were divided into: controls sedentary and trained receiving water, plant sedentary and trained receiving $104 \mathrm{mg} /$ day of $\mathrm{H}$. tomentosa infusion. The extensor digitorum longus muscle was harvested and used for histochemical, molecular and ultrastructural analysis. The main muscle fiber types were identified by m-ATPase technique. The point-sampling technique of classical stereology was employed to measure capillaries, muscular fiber and mitochondrial volume density. Testosterone levels and androgen receptor content increased significantly in plant sedentary animals. The muscle crosssection area of the plant trained animals increased significantly after training. Capillary and mitochondria volume density were significantly higher in the plant trained group compared to other groups. However, the association of $\mathrm{H}$. tomentosa and endurance training resulted in increased mean area of oxidative muscle fiber, capillary and mitochondrial volume density suggesting a potential increase of the endurance capacity of these animals.
\end{abstract}

Keywords: capillary density, EDL muscle, mitochondrial density, testosterone.

\section{Introduction}

The skeletal muscle is an extremely adaptable organ, demonstrating impressive structural and functional plasticity in response to alterations in metabolic and functional demand [1]. The muscle fiber types, which can be delineated according to various parameters such as myofibrillar protein isoforms, metabolic enzyme profiles, and structural and contractile properties, respond to altered functional demands and a variety of signals by changing their phenotypic profiles. These are affected mainly by innervations/neuromuscular activity, mechanical loading/unloading exercise, training, hormones, and aging [2] It is well established that increased contractile activity that occurs in endurance training promotes a transition from type II to type I muscle fiber types, which happens at the expense of the type II fiber population [3]. Moreover, endurance exercise increases mitochondrial density, capillary supply, changes in key metabolic enzymes, and increases maximal oxygen uptake [4]. In males, an acute bout of endurance exercise increases testosterone and dehydroepiandrosterone circulation [5,6] similar to the influence that regular exercise exerts on the resting hormone profile [7].

Heteropterys tomentosa A. Juss. (Malpighiaceae), previously known as Heteropterys aphrodisiaca, is found in the Cerrado biome of Mato Grosso and Goiás States (Central Brazil), and is commonly known as "nó-de-cachorro" [8,9]. It was described by Hoehne, in 1920, as a plant with stimulant and aphrodisiac properties and has been used in Brazilian traditional medicine for the treatment of nervous debility, nervous breakdown, and for muscle and bone weakness [8,9]. H. tomentosa roots contain anthracene and steroidal substances, along with tannins and high levels of flavonoids [10,11].

Previous studies suggested that the root extract could increase body and testicular weight along with the spermatogenic yield $[12,13]$. In addition, the association of $H$. tomentosa infusion with endurance training resulted in tibiae with higher yield load and yield stress [14]. This association contributed to more efficient remodeling of the extracellular matrix, resulting in more resistant tendons to support high loads from intense muscle contraction [15]. Therefore, we hypothesized that the endurance training would affect the muscle phenotype, and that the plant infusion could have a modulating role on these effects. We analyzed the plasma testosterone level, the concentration of androgen receptor in muscle, fiber composition, cross-section area of fibers, capillary density and mitochondrial density of the extensor digitorum longus muscle.

2.1 Animals

\section{Material and Methods}

Adult Wistar rats (Rattus norvegicus), 90 days old, were obtained from the Center for Biological Investigation CEMIB from the State University of Campinas (Campinas, SP, Brazil). Three animals were housed per cage under standard conditions with $12 \mathrm{~h}: 12 \mathrm{~h}$ light and dark cycles. Animals were provided with commercial rat feed and water ad libitum. This 
study was approved following the guidelines from the Institutional Committee for Ethics in Animal Care and Use (UNICAMP - protocol number 1233-1).

\subsection{Medicinal plant}

The plant roots were harvested in Mato Grosso State Brazil and identified by comparison with the voucher herbarium specimen located on the Federal University of Mato Grosso Herbarium collection, Brazil (number 23928). Briefly, the dried roots were crushed and powdered using a grinding mill. The infusion was routinely prepared as described previously [12-15]. The resulted infusion contained $68.66 \mathrm{mg}$ of dry extract $(6.866 \% \mathrm{w} / \mathrm{v})$ and a yield of $6.832 \%(\mathrm{w} / \mathrm{w})$ in terms of initial crude dry weight of plant material. The animals were weighed weekly to adjust the infusion dose.

\subsection{Study groups and experimental protocol}

Twenty-four male rats were divided into four groups ( $\mathrm{n}=6 /$ group): control sedentary $(\mathrm{CS}) ; H$. tomentosa sedentary (HS); control trained (CT); H. tomentosa trained (HT). The HS and HT received H. tomentosa infusion (104 mg/animal) daily, during the 8 weeks of training or sedentary period, whereas the control groups (CS and CT) received $0.5 \mathrm{~mL}$ of water, all of them via gavage. Trained rats (CT and HT groups) were allowed to adapt to treadmill running for a 3 week period, prior to the beginning of the experimental protocol, which consisted of low to moderate level of exercise carried out daily for 5 days a week. After adaptation, trained rats were subjected to 8 weeks of intensive aerobic exercise training (treadmill running), also on a weekly basis of 5 consecutive days followed by two days of rest, protocol adapted from [16].

\subsection{Surgical procedures}

Forty-eight hours after the last bout of training, the rats were anesthetized with xylazine chloride (Anasedan, Vetbrands, São Paulo, Brazil) and ketamine chloride (Cetamin, Syntec, Cotia, Brazil) (5 and $80 \mathrm{mg} / \mathrm{Kg}$ body weight, respectively). The blood was collected by heart puncture. The extensor digitorum longus muscles (EDL) were dissected, weighed and either frozen for histochemical, biochemical and Western blotting analysis (right hind leg) or preserved in Karnovsky's fixative for stereological analysis (left hind leg).

\subsection{Transmission electron microscopy}

Muscle fragments were fixed with Karnovsky's solution for 24 hours, post fixed with $1 \%$ osmium tetroxide (at $4^{\circ} \mathrm{C}$ ), dehydrated in acetone and embedded in epoxy resin (EMS 812). Ultra-thin sections (20-60nm) were stained with $2 \%$ uranyl acetate $(25 \mathrm{~min})$ and $2 \%$ lead citrate $(7 \mathrm{~min})$ prior to observation with a transmission electron microscope (Zeiss, Leo 906).

\subsection{Hormonal analysis}

Blood samples were centrifuged at 5,000 rpm for $10 \mathrm{~min}$ at $4^{\circ} \mathrm{C}$. The plasma was collected and the total testosterone was measured by chemi-luminescence in the Alvet Laboratory (Sorocaba, SP, Brazil).

\subsection{Western blotting}

Muscle samples were weighed and homogenized for $1 \mathrm{~min}$ with a Polytron homogenizer (Kinematica, Lucerne, Switzerland) in $200 \mu \mathrm{L}$ of a lysis buffer containing $150 \mathrm{mM} \mathrm{NaCl}, 1 \%$ Triton X-100, $10 \mathrm{mM}$ Tris pH 7.4, $1 \mathrm{mM}$ EDTA, $1 \mathrm{mM}$ EGTA, $1 \mathrm{mM}$ Hepes $\mathrm{pH} 7.6,2 \mathrm{mM}$ sodium vanadate, 0.2mM PMSF, 2mg/ml leupeptin, 2mg/ml aprotinin [17] and centrifuged at $10,000 \mathrm{xg}$ for $10 \mathrm{~min}$.

Protein concentration in the supernatant was determined using Bradford's reagent (Bio-Rad Laboratories,

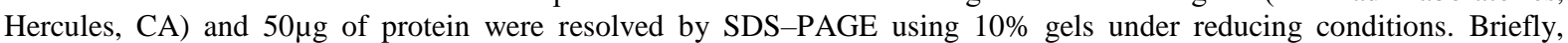
proteins were electro-transferred to nitrocellulose membranes (Hybond-ECL, Amersham Biosciences), which were subsequently blocked with TBS-T containing 5\% non-fat milk and probed with the antibody anti-androgen receptor (Cat. 06680; Millipore) diluted at 1:500 in TBS-T containing 1\% non-fat milk, followed by HRP-conjugated goat anti-rabbit IgG (Cat. A- 6154; Sigma Chemical Co.). The bands were developed using enhanced chemioluminescent substrate (Santa Cruz Biotechnology) and Kodak X-Omat films. The gels were stained with Coomassie Blue and the band corresponding to the molecular weight of tubulin $(55 \mathrm{kDa})$ was quantified to verify the quantity of protein loaded in the gels. Quantification of the AR bands was made by measuring the optical densities using the Scion Image software version 4.0.

\subsection{Determination of fiber type and morphometry}

The middle portion of the muscle was separated, oriented in a mixture of tragacanth gum (Sigma-G1128) and Tissue-Tek embedding compound (EMS, cat. 62550-01), immediately frozen in isopentane, cooled to $-156^{\circ} \mathrm{C}$ in liquid nitrogen, and stored at $-70^{\circ} \mathrm{C}$ until ready to use. Cross sections $(12 \mu \mathrm{m}$ thick) were obtained in a cryostat, collected on coverslips and stored at $-40^{\circ} \mathrm{C}$ until processing. Fiber types were identified using the myofibrillar adenosine triphosphatase (mATPase) identification following incubation at different pH: 4.2, 4.5 [18] and 10.6 [19] (Fig. 1). Three digital frames of each muscle section, obtained at $\mathrm{pH} 4.5$, were selected, according to [20]. These were photographed and mounted as a plate. This plate was used as a guide to identify the pure fiber types (I, IIA, IIB and IID) from sections obtained at pH 4.2, 4.5 and 10.6. Image Pro-Plus software (Media Cybernetics, Inc.) was used to calculate the cross-sectional areas of muscle fibers using a BX50 Olympus light microscope. The same three frames used for fiber-type determination were used and all muscle fibers within these fields were evaluated.

\subsection{Stereology}

Capillaries were identified in the same cross section fields used for fiber-type determination. The number of capillaries per unit area (capillary density, $\mathrm{CD}=$ capillaries $/ \mathrm{mm} 2$ ) was measured. The capillary and fiber densities were 
obtained by the stereological methods described previously [21]. The stereological analysis used a test-system with 112-testpoints in a known area. The volume densities of the structures were estimated as (1) Vv[structure]= PP[structure]/PT, where PP is the number of points that were superimposed over the structure and PT is the total number of test-points contained in the area surrounded by the frame. Muscular vascularization was calculated by the ratio of capillary volume density and fiber volume density [22].

The number and volume of mitochondria were determined using electron micrographs of the fibers at $10,000 \mathrm{x}$ magnification. All mitochondria were counted in each image, whereas their volume density was calculated using a 140 testpoint-grid [21]. The results were expressed as the ratio of mitochondrial volume density and mean fiber cross-section area.

\subsection{Statistics}

The statistical analyses were performed using the software Statistica (v 8.0) (Tulsa, OK, USA). All data were presented as mean \pm SD and the significance level was set as $5 \%$. The statistical comparisons among control and treated groups were made using one-way ANOVA followed by the post hoc test of Duncan. In addition, two-way ANOVA was used, when appropriate, to determine how $H$. tomentosa treatment and/or exercise training affected the results, and whether there was interaction between these two conditions.

\section{Results}

\subsection{Biometry and hormonal analysis}

Body mass increased in all groups during the treatment. Both trained groups gained less weight than the sedentary ones, although there were no alterations of the EDL weight (Table 1). The sedentary animals treated with H. tomentosa (HS) showed the highest testosterone levels (Table 1).

Table 1. Biometry and testosterone dosage of rats treated with $H$. tomentosa and submitted to treadmill training.

\begin{tabular}{|l|c|c|c|c|}
\hline Parameter & Control sedentary & $\begin{array}{c}\text { H. tomentosa } \\
\text { sedentary }\end{array}$ & Control trained & H. tomentosa trained \\
\hline Body mass gain $(\mathrm{g})$ & $72.00 \pm 12.41$ & $68.5 \pm 15.54$ & $57.6 \pm 12.02$ & $61.00 \pm 16.57$ \\
\hline EDL (mg) & $167 \pm 19.00$ & $183 \pm 22.00$ & $165 \pm 25.00$ & $180 \pm 18.00$ \\
\hline Relative weight EDL & $0.040 \pm 0.004$ & $0.044 \pm 0.005$ & $0.041 \pm 0.005$ & $0.041 \pm 0.005$ \\
\hline Testosterone (ng/mL) & $3.28 \pm 0.894$ & $5.13 \pm 0.614^{*}$ & $3.08 \pm 0.353$ & $2.51 \pm 0.55$ \\
\hline
\end{tabular}

Values are mean \pm SD. $* \mathrm{p}<0.05$ (ANOVA and Duncan test).

\subsection{Western blotting}

The Western blotting analysis showed a significant increase in the AR protein concentration in EDL muscle of HS group (Fig. 2).

\subsection{Fiber type and morphometry}

The fiber types phenotype did not change among groups (Fig. 3a). However, we observed a trend towards the transformation of the IIB $\rightarrow$ IIA fiber types in the trained groups. The fiber's area was not altered after the plant intake, however the association of the infusion with the endurance training induced a positive effect, with the highest mean cross-sectional area of muscle fibers ( $\mathrm{p}=0.032)$ (Fig. 3b).

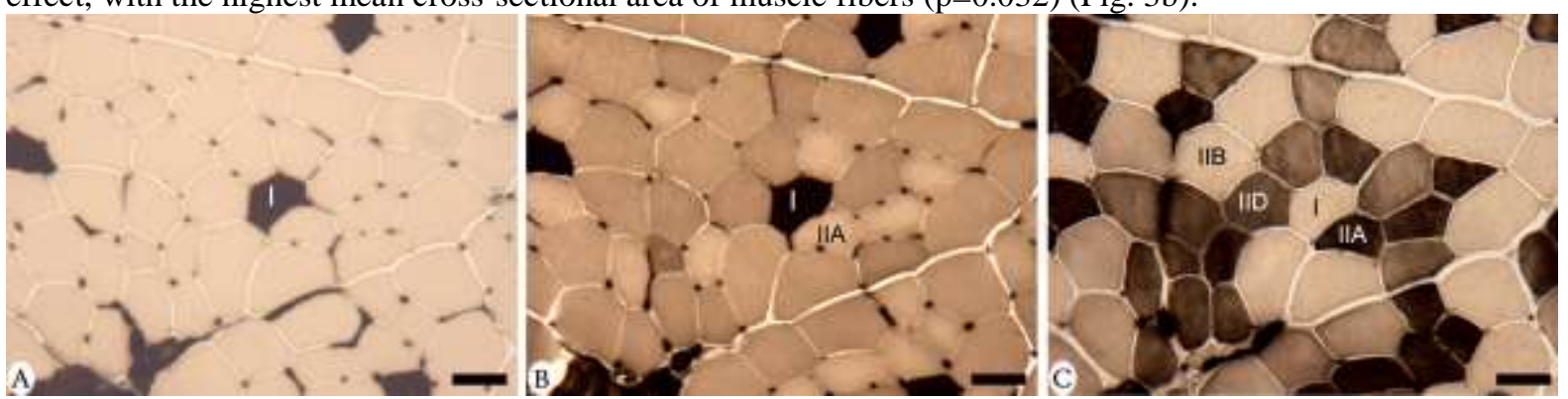

Fig. 1 Histochemical mATPase reaction at pH 4.2 (a), pH 4.5 (b) and $\mathrm{pH} 10.6$ (c) in EDL muscles sections. I type I fiber, IIA - type IIA fiber, IIB - type IIB fiber, and IID - type IID fiber. Bar: $20 \mu \mathrm{m}$. 

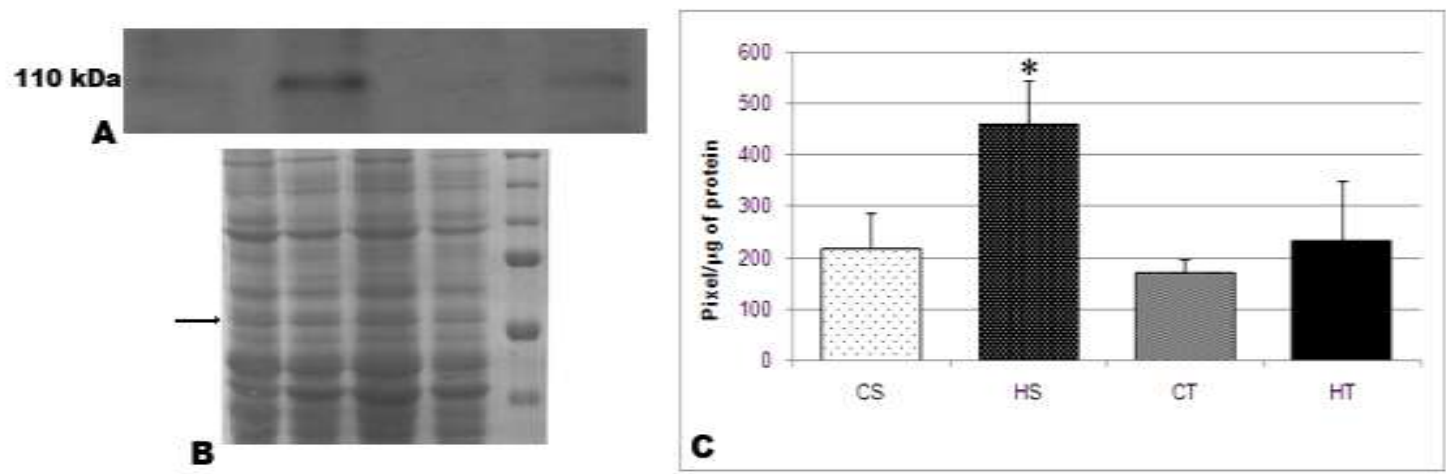

Fig. 2 Western blot analysis of androgen receptor (AR) in the EDL muscle. a Representative blot showing AR levels in EDL muscle. b Coomassie blue-stained membrane demonstrates even loading. $\mathbf{c}$ AR concentration in different groups. CS- Control sedentary, HS- H. tomentosa sedentary, CT-Control trained, HT- H. tomentosa trained. In $\mathrm{C}$ the values are mean $\pm \mathrm{SD}$. ${ }^{*} \mathrm{p}<0.05$ (ANOVA, Duncan test). Arrow- Bands corresponding to tubulin $(55 \mathrm{kDa})$.

\subsection{Stereology}

The capillary volume density increased in the HT group $(\mathrm{p}<0.05)$. No alterations were observed in the capillary and mitochondria numbers and the fiber volume density (data not shown). The intramuscular vascularization increased after training, however it was significantly higher in HT group compared with the CS (Fig. 4a). The mitochondria volume density was significantly higher in HT group (Fig. 4b).
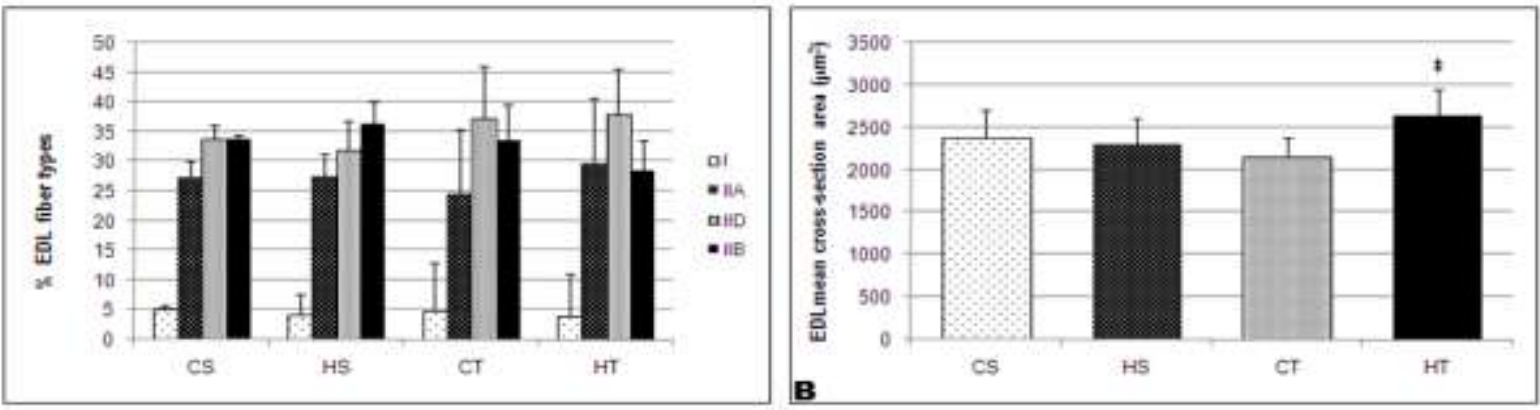

Fig. 3 Percentage of different fiber types (a) and mean cross-section area (b) of EDL. CS- control sedentary; HS- $H$. tomentosa sedentary; CT- control trained; HT- H. tomentosa trained. The columns are the mean \pm SD. $\ddagger$ $\mathrm{p}<0.05$ (ANOVA, Duncan test) when compared with CT group.
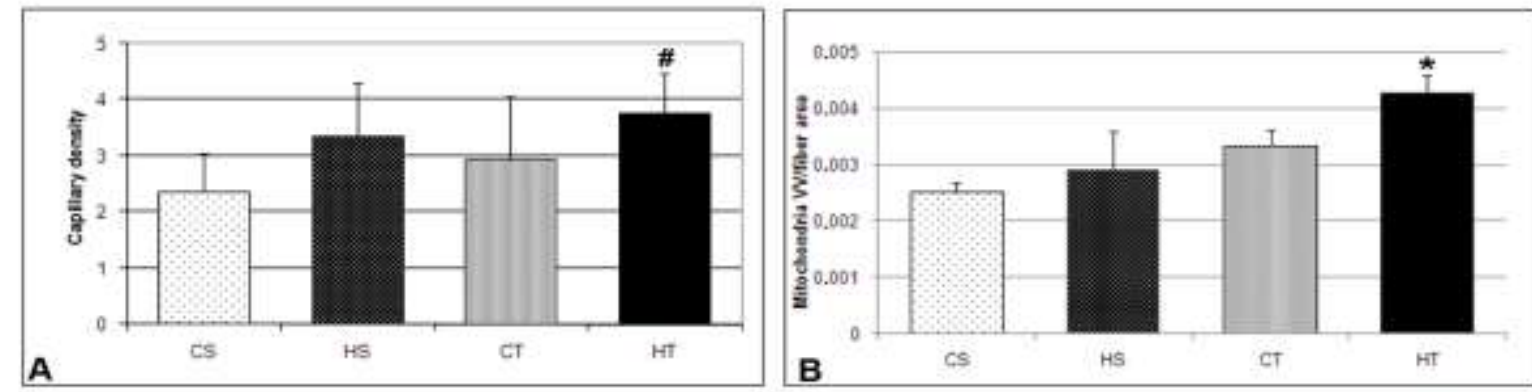

Fig. 4 Effect of $H$. tomentosa infusion and endurance training on muscle capillaries and mitochondrial content. a Capillary density (ratio of capillary volume density and fiber volume density); b Ratio of mitochondria volume density and mean fiber cross-section area. CS- Control sedentary, HS- H. tomentosa sedentary, CT- Control trained, HT- H. tomentosa trained. Values are mean \pm SD. ${ }^{\#} \mathrm{p}<0.05$ (ANOVA, Duncan test) when compared with CS group. * $\mathrm{p}<0.05$ (ANOVA, Duncan test). 


\section{Discussion}

This study was designed to verify the effect of $H$. tomentosa upon the skeletal muscle fibers of adult male Wistar rats submitted to endurance training. We evaluated alterations of testosterone levels, the expression of androgen receptors, the types and ultrastructure of fibers from the extensor digitorum longus muscle from animals treated with the plant infusion and submitted or not to the endurance training. Many authors have investigated the effect of exercise in the endocrine system. While some studies demonstrated that endurance training does not lead to lower total testosterone levels in athletes $[23,24]$, others have clearly shown that hormone levels vary during training [25-27]. These contradictory results can be explained by the dependence of such reactions on many different factors such as intensity, duration and type of exercises, as well as the level of the athletes' training [26].

The long term intake of $H$. tomentosa infusion significantly increased serum testosterone levels even in sedentary animals, the same behavior observed for the androgen receptors (AR) concentration. These findings explain the higher weight of the prostate and seminal vesicles, as well as the higher spermatogenic yield described by Gomes et al. [13] for the same animals. Interestingly, increasing of hormonal parameters did not induce muscle mass gain in response to the exercise load, as it was expected [28,29]. The association between plant infusion and physical exercise did not alter testosterone levels, as also occurred in control trained animals, which corroborates the results observed for athletes under endurance exercise $[23,30]$.

Endurance training can induce antagonistic stimulus to increase whole muscle cross-section area, and even a higher concentration of hormones and receptors [31]. Supporting this theory, molecular biology studies showed the concurrent adaptive mechanisms in skeletal muscle transcription signal pathways when different kinds of exercise stimulus where present [32]. Applying those concurrent training concepts in our results, it is possible to assume that our training program was able to induce classic aerobic adaptations, such as increased mean area of oxidative muscle fiber, capillary and mitochondrial volume density without increasing final muscle mass [33,34].

Endurance training can induce up-regulation of adenosine monophosphate-activated kinase $\alpha$-peroxisome proliferator activated receptor gamma coactivator-1 $\alpha$ (AMPK-PGC-1 $\alpha$ ) pathway, at the same time as it can down-regulate the Akt/protein kinase B-mammalian target of rapamycin-p70S6 kinase (Akt-mTOR-S6K) pathway, very much involved in strength training response [35]. This difference in signaling pathway activation could be one of the possible explanations for specific aerobic adaptations derived from our eleven week training program.

There is considerable evidence supporting that endurance training usually results in increased mitochondrial content and capillary density instead of altering whole muscle cross-section areas $[31,36]$. Considering the muscle crosssection area, the anabolic effect associated to the plant infusion administration was related to the most recruited fibers (type I), supporting the well described event of selective fiber hypertrophy [37]. Changes in the cross-section area of fibers indicate synthesis of contractile proteins, including myosin. It also shows that new myofibrils were added to the fiber with the synthesis of such proteins [38]. EDL cross sections analysis showed that some of the control trained animals had lower average areas, but comparing with sedentary animals this reduction was not significant. Therefore, the endurance training without infusion treatment did not cause alterations in the cross section area of the rats' muscle fibers, thus differing from data presented by Harber et al. [39] and Trappe et al. [40], who related reduction of fiber diameters after endurance training in athletes. These differences can be explained by the type and duration of the exercise protocols along with the differences between species. On the other hand, the association of the plant infusion and treadmill exercise resulted in a significant increase of the average cross section area of the muscle fibers, when compared to the control trained animals.

Testosterone directly stimulates muscle growth by affecting the rate of protein synthesis, protein breakdown, and net gain or loss of muscle. These actions are reportedly mediated by the androgen receptor, which acts as a nuclear transcription factor [41]. In addition, locally produced insulin-like growth factor 1 (IGF-1) was found to increase after acute muscle damage [42] or chronic aerobic exercise [43], with no changes in circulating IGF-1 [42,43]. On the other hand, the increase of serum IGF-1 with exogenous administration of growth hormone or IGF-1 does not appear to stimulate fiber hypertrophy in the absence of mechanical loading [44]. A sustained local overexpression of IGF-1 promotes fiber regeneration and hypertrophy through various pathways and has been shown to increase levels of myogenic regulatory factors and contractile protein mRNAs. IGF-1 also seems essential to mediate the loading-induced hypertrophy of skeletal muscle $[45,46]$. Based on this information, we believe that more studies are necessary to determine which molecules initiate muscle hypertrophy in trained animals treated with the plant infusion, since the increase of plasma testosterone did not alter muscle fiber morphometry.

It has been assumed that changes in the isoforms of myosin during endurance training can be characterized as quantitative remodeling of the muscle, in that one isoform is substituted by another which is more adapted to the level of strength needed for long duration exercise [47]. In some situations, endurance exercise promoted the transition from rapid to slower contracting fibers. This does not lead to a transformation of type II into type I, but a change within the type II fiber subtypes, in the direction of types IIB $\rightarrow$ IID $\rightarrow$ IIA $[4,48]$, although the type of change will depend on the analyzed muscle. Our findings did not show significant differences in the percentage of the muscle fiber types of the EDL of different experimental groups, despite the trend of increasing IID fibers and decreasing of IIB fibers, especially in trained groups. The tendency of fiber type transformation can reflect the greater oxidative potential in endurance trained animals [49]. Our results differ from the results of Seene and collaborators [4], which showed significant changes in the myosin heavy-chain isoforms after treadmill training. The association of infusion with endurance training led to an upgrade in EDL muscle fibers anabolism demonstrated by the larger cross-section area in relation to CT group. This effect is very interesting considering that the endurance training alone could be useful to promote selective fiber type hypertrophy [37], counterbalancing eventual muscle atrophy and sarcopenia often associated with age or post-immobilization [50,51]. On the other hand, endurance training could induce a decrease in different fiber type cross-section area and relative atrophy of whole muscles, mainly in fasted condition [40,52]. 
In this study, we found that the association of the plant infusion and endurance training increased volumetric density of mitochondria and capillaries, thus increasing both vascular and oxidative systems of the muscle tissue. However, endurance training alone was not enough to disturb such parameters, which is in disagreement with previous the results of, who demonstrated the increasing of volume density of mitochondria and/or capillaries in muscles of humans and rats after endurance training [53-56].

Therefore, the association of the plant infusion with endurance training could have induced capillary angiogenesis within and among skeletal muscles and, since the capillary and oxidative capacities are coupled [57], with the increased mitochondrial volume density that has been observed. Exercise induced increasing of mitochondrial volume was described first in 1967 [58]. Nowadays, some intracellular transcription factors have been pointed out to be responsible for this adaptive response, including nuclear respiratory factors 1 and 2 (NRF-1 and NRF-2) and peroxisome proliferator-activated receptor $\gamma$ coactivator- $1 \alpha$ (PGC-1 $\alpha$ ) [59,60]. The main signals for mitochondria biogenesis during endurance training are ATP and phosphocreatine decrease, as well as $\mathrm{Ca}^{+2}$ releases from sarcoplasmic reticulum during muscle contraction (E-C coupling) $[61,62]$.

\section{Conclusion}

The training protocol used in this study did not alter the muscle in control animals; however the association of $H$. tomentosa and the endurance training protocol resulted in increased mean muscle fiber area, mitochondrial volume density and muscular vascularization. Thus, it can be concluded that the plant infusion and endurance training led to hypertrophy of oxidative muscle fibers and potentially improved the endurance capacity of animals trained and treated during eight weeks.

\section{Acknowledgements}

The authors wish to thank Taize Augusto for the Western Blotting analysis, Danilo Ferrucci for contributions to the above discussion and Karina Fontana for helping with the training protocol. This work was supported by grants from FAPESP (Fundação de Amparo à Pesquisa do Estado de São Paulo), Grant number: 2008/05610-3.

\section{References}

[1]. R.T. Hepple, Skeletal muscle: microcirculatory adaptation to metabolic demand, Medicine \& Science in Sports \& Exercise, 32: 2000, 117123.

[2]. D. Pette and R.S. Staron, Transitions of muscle fiber phenotypic profiles, Histochemistry and Cell Biology, 115: 2001, 359-372.

[3]. R. Thayer, J. Collins, E.G. Noble and A.W. Taylo, Histological evidence for fibre type transformation, The Journal of Sports Medicine and Physical Fitness, 40(4): 2000, 284-9.

[4]. T. Seene, K. Alev, P. Kaasik, A. Pehme and A.M. Parring, Endurance training: volume-dependent adaptation changes in myosin, International Journal of Sports \& Medicine, 26: 2005, 815-821.

[5]. H. Galbo, L. Hummer, B. Petersen, N.J. Christensen and N. Bie, Thyroid and testicular hormone responses to graded and prolonged exercise in man, European Journal of Applied Physiology, 36: 1977, 101-106.

[6]. M.S. Tremblay, J.L. Copeland and W. Van Helder, Influence of exercise duration on post-exercise steroid hormone responses in trained males, European Journal of Applied Physiology, 94: 2005, 505-513.

[7]. A.C. Hackney, C.L. Fahrner and T.P. Gulledge, Basal reproductive hormonal profiles are altered in endurance trained men, The Journal of Sports Medicine and Physical Fitness, 38: 1998, 138-141.

[8]. M. Pio Corrêa, Dicionário de Plantas Úteis do Brasil e das Exóticas Cultivadas (Ministério da Agricultura, Brasília: Instituto Brasileiro de Desenvolvimento Florestal, 1984).

[9]. A. Pott and V.J. Pott, Plantas do Pantanal (Corumbá: Empresa Brasileira de Pesquisa Agropecuária do Pantanal, 1994).

[10]. S.M.P. Galvão, L.C. Marques, M.G.M. Oliveira and E.A. Carlini, Heteropterys tomentosa (extract BST0298): a Brazilian plant that improves memory in aged rats, Journal of Ethnopharmacology, 79: 2002, 305-311.

[11]. L.C. Marques, C. Pieri, W.A. Roman-Júnior, M.L.C. Cardoso, M.A. Milaneze-Gutierre and J.C.P. Mello, Controle farmacognóstico das raízes de Heteropteris aphrodisiaca O. Mach. (Malpighiaceae), Revista Brasileira de Farmacognosia, 17: 2007, 604-615.

[12]. J.C. Monteiro, F.S. Predes, S.L. Matta And H. Dolder, Heteropterys aphrodisiaca infusion reduces the collateral effects of cyclosporine A on the testis, Anatomical Records (Hoboken), 291: 2008, 809-817.

[13]. M.L.M. Gomes, J.C. Monteiro, M.M. Sbervelheri and H. Dolder, Association of the infusion of Heteropterys aphrodisiaca and endurance training brings spermatogenetic advantages, Biological Research, 44: 2011, 235-241.

[14]. J.C. Monteiro, M.L.M. Gomes, W.R. Nakagaki, T.C. Tomiosso, M.M. Sbervelheri, and H. Dolder, Does Heteropterys aphrodisiaca administration and endurance training alter bones of mature rats?, Journal of Morphoogical Science, 27: 2010, 67-73.

[15]. J.C. Monteiro, M.L.M. Gomes, T.C. Tomiosso, W.R. Nakagaki, M.M. Sbervelheri, D.L. Ferrucci, E.R. Pimentel and H. Dolder, More resistant tendons obtained from the association of Heteropterys aphrodisiaca and endurance training, BMC Complementary and Alternative Medicine, 11: 2011, 51 .

[16]. H.A. Demirel, S.K. Powers, M.A. Zergeroglu, R.A. Shanely, K. Hamilton, J. Coombes and H. Naito, Short-term exercise improves myocardial tolerance to in vivo ischemia-reperfusion in the rat, Journal of Applied Physiology, 91: 2001, 2205-2212.

[17]. W.F. Zambuzzi, J.M. Granjeiro, K. Parikh, S. Yuvaraj, M.P. Peppelenbosch. and C.V. Ferreira, Modulation of Src activity by low molecular weight protein tyrosine phosphatase during osteoblast differentiation, Cellular Physiology and Biochemistry, 22: 2008, 497-506.

[18]. R.S. Staron and D. Pette, The continuon of pure and hybrid myosin heavy chain-based fiber types in rat skeletal muscle, Histochemistry, 100: 1993, 149-153.

[19]. L. Guth and F.J. Samaha, Procedure for the histochemical demonstration of actomyosin ATPase, Experimental Neurology, 28: 1970, 365367.

[20]. S. Esteva, P. Panisello, M. Casas, J.R. Torrella, T. Pagés and G. Viscor, Morphofunctional responses to anaemia in rat skeletal muscle, Journal of Anatomy, 212: 2008, 836-844.

[21]. E.R. Weibel, Stereological methods. I. Practical methods for biological morphometry. (London: Academic Press, 1979).

[22]. C.A. Mandarim-De-Lacerda, Stereological tools in biomedical research, Anais da Academia Brasileira de Ciências, 75: 2003, 469-486.

[23]. A. Lucia, J.L. Chicharro, M. Pérez, L. Serratosa, F. Bandrés and J.C. Legido, Reproductive function in male endurance athletes: sperm analysis and hormonal profile, Journal of Applied Physiology, 81: 1996, 2627-2636.

[24]. I. Smilios, T. Pilianidis, M. Karamouzis and S.P. Tokmakidis, Hormonal responses after various resistance exercise protocols, Medicine \& Science in Sports \& Exercise, 35: 2003, 644-654.

[25]. G.D. Wheeler, M. Singh, W.D. Pirce, W.F. Epling and D.C. Cumming, Endurance training decreases serum testosterone levels in men without change in luteinizing hormone pulsatile release, The Journal of Clinical Endocrinology \& Metabolism, 72: 1991, 422-425. 
[26]. M.S. Tremblay, J.L. Copeland and W. Van Helder, Effect of training status and exercise mode on endogenous steroid hormones in men, Journal of Applied Physiology, 96: 2004, 531-539.

[27]. P. Sgrò, F. Romanelli, F. Felici, M. Sansone, S. Bianchini, C.F. Buzzachera, C. Baldari, L. Guidetti, F. Pigozzi, A. Lenzi and L. Di Luigi, Testosterone responses to standardized short-term sub-maximal and maximal endurance exercises: issues on the dynamic adaptive role of the hypothalamic-pituitary-testicular axis, Journal of Endocrinological Investigation, 37: 2014, 13-24

[28]. M.R. Deschenes, C.M. Maresh, L.E. Armstrong, J. Covault, W.J. Kraemer and J.F. Crivello, Endurance and resistance exercise induce muscle fibre type specific responses in androgen binding capacity, The Journal of Steroid Biochemistry and Molecular Biology, 50: 1994, 175-179.

[29]. F. Kadi, Adaptation of human skeletal muscle to training and anabolic steroids, Acta Physiologica Scandinavica, 168: 2000, 44-53

[30]. M. Duclos, J.B. Corcuff, M. Rashedi, V. Fougere and G. Manier, Does functional alteration of the gonadotropic axis occur in endurance trained athletes during and after exercise? A preliminary study, European Journal of Applied Physiology and Occupational Physiology, 73: 1996, 101-106.

[31]. L. Karavirta, K. Häkkinen, A. Kauhanen, A. Arija-Blázquez, E. Sillanpää, N. Rinkinen and A. Häkkinen, Individual responses to combined endurance and strength training in older adults. Medicine \& Science in Sports \& Exercise, 43: 2011, 484-490.

[32]. R.C. Hickson, M.A. Rosenkoetter and M.M. Brown, Strength training effects on aerobic power and short-term endurance, Medicine \& Science in Sports \& Exercise, 12: 1980, 336-339.

[33]. I. Irrcher, P.J. Adhihetty, A.M. Joseph, V. Ljubicic and D.A. Hood, Regulation of Mitochondrial Biogenesis in Muscle by Endurance Exercise, Sports Medicine, 33: 2003, 783-793.

[34] M. Gibala, Molecular responses to high-intensity interval exercise, Applied Physiology and Nutrition Metabolism, 34: 2009, 428-432.

[35]. V.G. Coffey, H. Pilegaard, A.P. Garnham, B.J. O'brien and J.A. Hawley, Consecutive bouts of diverse contractile activity alter acute responses in human skeletal muscle, Journal of Applied Physiology, 106: 2009, 1187-1197.

[36]. J.O. Holloszy and E.F. Coyle, Adaptations of skeletal muscle to endurance exercise and their metabolic consequences, Journal of Applied Physiology, 56: 1984, 831-838.

[37]. J.A. Hawley, Adaptations of skeletal muscle to prolonged, intense endurance training, Clinical and Experimental Pharmacology and Physiology, 29: 2002, 218-222.

[38]. F. Booth, B. Tseng, M. Fluck and J. Carson, Molecular and cellular adaptation of muscle in response to physical training, Acta Physiologica Scandinavica, 162: 1998, 343-350.

[39]. M.P. Harber, P.M. Gallsgher, A.R. Creer, K.M. Minchev and S.W. Trappe, Single muscle fiber contractile properties during a competitive season in male runners, The American Journal of Physiology - Regulatory, Integrative and Comparative Physiology, 287: 2004, R1124R1131.

[40]. S. Trappe, M. Harber, A. Creer, P. Gallagher, D. Slivka, K. Minchev and D. Whitsett, Single muscle fiber adaptations with marathon training, Journal of Applied Physiology, 101: 2006, 721-727.

[41]. F. Hartgens and H. Kuipers, Effects of androgenic-anabolic steroids in athletes, Sports Medicine, 34: 2004, 513-554

[42]. M.M. Bamman, J.R. Shipp, J. Jiang, B.A. Gower, G.R. Hunter, A. Goodman, C.L. Mclafferty and R.J. Urban, Mechanical load increases muscle IGF-1 and androgen receptor mRNA concentrations in humans, American Journal of Physiology and Endocrinology Metabolism, 280: 2001, E383-E390.

[43]. R. Hambrecht, P.C. Schulze, S. Gielen, A. Linke, S. Mobius-Winkler, S. Erbs, J. Kratzsch, A. Schubert, V. Adams and G. Schuler, Effects of exercise training on insulin-like growth factor-I expression in the skeletal muscle of non-cachectic patients with chronic heart failure, European Journal of Cardiovascular Prevention \& Rehabilitation, 12: 2005, 401-406.

[44]. M.M. Bamman, M.S. Clarke, D.L. Feeback, R.J. Talmadge, B.R. Stevens, S.A. Lieberma and M.C. Greenisen, Impact of resistance exercise during bed rest on skeletal muscle sarcopenia and myosin isoform distribution, Journal of Applied Physiology, 84: 1998, 157-163.

[45]. A. Musaro, K. Mccullagh, A. Paul, L. Houghton, G. Dobrowolny, M. Molinaro, E.R. Barton, H.L. Sweeney and N. Rosenthal, Localized IGF-1 transgene expression sustains hypertrophy and regeneration in senescent skeletal muscle, Nature Genetics, 27: 2001, 195-200.

[46]. J.L. Olesen, K.M. Heinemeier, F. Haddad, H. Langberg, A. Flyvbjerg, M. Kjaer and K.M. Baldwin, Expression of insulin-like growth factor I, insulin-like growth factor binding proteins, and collagen mRNA in mechanically loaded plantaris tendon, Journal of Applied Physiology, 101: 2006, 183-188

[47]. K.M. Baldwin and F. Haddad, Effects of different activity and inactivity paradigms on myosin heavy chain gene expression in striated muscle, Journal of Applied Physiology, 90: 2001, 345-357.

[48]. T. Okumoto, T. Omoto, S. Katsuta and M. Wada, Severe endurance training fails to change myosin heavy-chain distribution of diaphragm, Respiratory Physiology, 104: 1996, 39-43.

[49]. P. Diaz-Herrera, J.M. García-Castellano, A. Torres, J.A. Morcuende, J.A. Calbet and R. Sarrat, Journal of Orthopaedic Research, 19: 2001, 229-232.

[50]. Y. Boirie, Physiopathological mechanism of sarcopenia, Journal of Nutrition Health and Aging 13: 2009, 717-723.

[51]. T. Gwag, K. Lee, H. Ju, H. Shin, J.W. Lee and I. Choi, Stress and signaling responses of rat skeletal muscle to brief endurance exercise during hindlimb unloading: a catch-up process for atrophied muscle, Cellular Physiology and Biochemistry, 24: 2009, 537-546.

[52]. W.J. Kraemer, J.F. Patton, S.E. Gordon, E.A. Harman, M.R. Deschenes, K. Reynolds, R.U. Newton, N.T. Triplett and J.E. Dziados, Compatibility of high-intensity strength and endurance training on hormonal and skeletal muscle adaptations, Journal of Applied Physiology, 78: 1995, 976-989.

[53]. H. Hoppeler, H. Howald, K. Conley, S.L. Lindstedt, H. Claassen, P. Vock and E.R. Weibel, Endurance training in humans: aerobic capacity and structure of skeletal muscle, Journal of Applied Physiology, 59: 1985, 320-327.

[54]. H. Howald, H. Hoppeler, H. Claassen, O. Mathieu-Costello and R. Straub, Influences of endurance training on ultrastructural composition of the different muscle fiber types in humans, Pflugers Archiv-European Journal of Physiolgy, 403: 1985, 369-376.

[55]. D.C. Poole and O. Mathieu-Costello, Relationship between fiber capillarization and mitochondrial volume density in control and trained rat soleus and plantaris muscles, Microcirculation, 3: 1997, 175-186.

[56]. T.P. Gavin, R.S. Ruster, J.A. Carrithers, K.A. Zwetsloot, R.M. Kraus, C.A. Evans, D.J. Knapp, J.L. Drew, J.S. Mccartney, J.P. Garry and R.C. Hickner, No difference in the skeletal muscle angiogenic response to aerobic exercise training between young and aged men, Journal of Physiology 585: 2007, 231-239.

[57]. V.A. Lira, C.R. Benton, Z. Yan and A. Bonen, PGC-1 $\alpha$ regulation by exercise training and its influences on muscle function and insulin sensitivity, American Journal of Physiology and Endocrinology Metabolism, 299: 2010, E145-E161.

[58]. J.O. Holloszy, Biochemical adaptations in muscle. Effects of exercise on mitochondrial oxygen uptake and respiratory enzyme activity in skeletal muscle, Journal of Biological Chemistry, 242: 1967, 2278-2282.

[59]. R.C. Scarpulla, Nuclear activators and coactivators in mammalian mitochondrial biogenesis. Biochimica et Biophysica Acta, 1576: 2002, 114.

[60]. J. Lin, C. Handschin and B.M. Spiegelman, Metabolic control through the PGC-1 family of transcription coactivators, Cell Metabolism, 1: $2005,361-70$

[61]. J.O. Holloszy, Regulation by exercise of skeletal muscle content of mitochondria and GLUT4, Journal of Physiology and Pharmacology, 59: 2008, 5-18.

[62]. P.J. Fernandez-Marcos and J. Auwerx, Regulation of PGC-1 $\alpha$, a nodal regulator of mitochondrial biogenesis, American Journal of Clinical Nutrition, 93: 2011, 884S-890. 